\title{
Profil complet des caractéristiques sociodémographiques, psychosociales et sanitaires des clients des soins à domicile atteints de démence en Ontario
}

M. Vu, M. Sc. (1, 2); D. B. Hogan, M.D. (3, 4); S. B. Patten, M.D., Ph. D. (4); N. Jetté, M.D., M. Sc. (4, 5);

S. E. Bronskill, Ph. D. (6, 7); G. Heckman, M.D. (2); M. J. Kergoat, M.D. (8); J. P. Hirdes, Ph. D. (2); X. Chen, M. Sc. (2); M. M. Zehr, B. Sc. (1); C. J. Maxwell, Ph. D. $(1,2,4,6)$

Cet article a fait l'objet d'une évaluation par les pairs.

\section{Résumé}

Introduction : Cette étude fournit une synthèse des caractéristiques sociodémographiques, psychosociales et sanitaires d'une vaste cohorte représentative des clients des soins à domicile en Ontario (âgés de 50 ans ou plus) atteints de démence et elle examine les variations de ces caractéristiques chez les clients atteints de maladies neurologiques concomitantes.

Méthodologie : Les clients ont été évalués à l'aide de l'Instrument d'évaluation des résidents - Soins à domicile (RAI-HC) entre janvier 2003 et décembre 2010. Les analyses descriptives fournissent la répartition de ces caractéristiques en comparant les clients atteints de démence et ceux de plusieurs autres groupes ainsi que ceux atteints d'autres maladies neurologiques documentées.

Résultats : Environ $22 \%$ des clients $(n=104$ 802) avaient reçu un diagnostic de démence (âge moyen de 83 ans, $64 \%$ de femmes) et un sur quatre parmi eux était atteint d'une maladie neurologique concomitante (AVC ou maladie de Parkinson la plupart du temps). Environ $43 \%$ des clients atteints de démence n'habitaient pas avec leur principal aidant. Par rapport aux clients des groupes de comparaison, les clients atteints de démence présentaient des taux considérablement plus élevés de déficit cognitif et fonctionnel, d'agressivité, d'anxiété, d'errance et d'hallucinations ou de délire, avaient plus souvent un aidant en détresse et couraient un plus grand risque de placement en établissement. Par contre, ils étaient moins souvent atteints de diverses maladies chroniques et étaient moins nombreux à avoir eu recours à des services de santé récemment. Les symptômes de dépression étaient relativement fréquents chez les clients atteints de démence et chez ceux atteints d'une autre maladie neurologique.

Conclusion : Les clients atteints de maladies neurologiques concomitantes présentaient des profils cliniques bien particuliers illustrant la nécessité de personnaliser et d'assouplir les services de soins à domicile et d'améliorer les programmes de soutien pour les aidants.

Mots-clés : démence, maladie d'Alzheimer, troubles neurologiques, santé mentale, soins à domicile

\section{Introduction}

On estime qu'à l'heure actuelle environ 35,6 millions de personnes sont atteintes d'une forme de démence, dont la maladie d'Alzheimer, à l'échelle mondiale ${ }^{1}$. Au Canada, environ un demi-million de personnes sont atteintes de démence et, selon les estimations, la prévalence augmente de façon exponentielle après 65 ans $^{2}$. Outre le coût personnel de la maladie, la prestation de soins continus aux personnes atteintes de démence représente un fardeau sociétal et économique important tant du point de vue des soins prodigués par la famille que du point de vue des services de soins professionnels et des coûts qui y sont associés $^{3-6}$. Alors que relativement peu de personnes âgées ont besoin de soins coûteux en établissement en vieillissant ${ }^{7}$, le risque augmente considérablement chez les aînés atteints de démence ${ }^{8}$. La prestation de services de soins à domicile coordonnés, appropriés et fournis en temps opportun aux aînés canadiens atteints de démence peut contribuer à réduire les risques et les coûts associés aux soins en établissement tout en prenant en compte les préférences des personnes âgées qui souhaitent demeurer plus longtemps chez elles, dans un cadre familier et à proximité de leurs réseaux sociaux? .

Rattachement des auteurs :

1. École de pharmacie, Université de Waterloo, Waterloo (Ontario), Canada

2. École de la santé publique et des systèmes de santé, Université de Waterloo, Waterloo (Ontario), Canada

3. Division de médecine gériatrique, Faculté de médecine, Université de Calgary, Calgary (Alberta), Canada

4. Département des sciences de la santé communautaire et Institut de santé publique, Université de Calgary, Calgary (Alberta), Canada

5. Département des neurosciences cliniques et Hotchkiss Brain Institute, Université de Calgary, Calgary (Alberta), Canada

6. Institut de recherche en services de santé, Toronto (Ontario), Canada

7. Institut des politiques, de la gestion et de l'évaluation de la santé, Université de Toronto, Toronto (Ontario), Canada

8. Centre de recherche, Institut universitaire de gériatrie de Montréal, Faculté de médecine, Université de Montréal, Montréal (Québec), Canada

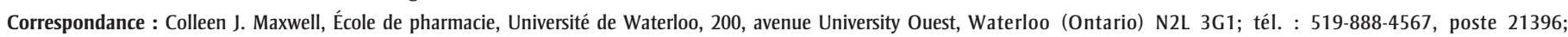
téléc. : 519-883-7580; courriel : colleen.maxwell@uwaterloo.ca 
Parmi le million de Canadiens qui, selon les estimations, reçoivent des soins à domicile à un moment ou à un autre ${ }^{10}$, plus des trois quarts (82\%) sont âgés de 65 ans ou plus ${ }^{11}$ et environ $20 \%$ sont atteints de la maladie d'Alzheimer ou d'une autre forme de démence ${ }^{12}$. Il est essentiel de bien connaître les besoins en matière de santé physique, mentale et sociale des aînés canadiens atteints de démence qui reçoivent des soins communautaires afin d'assurer une planification des soins adaptée à cette population vulnérable et en augmentation, ainsi qu'une prise en charge optimale. Un examen attentif des caractéristiques des clients et de leurs besoins en matière de soins pourrait aussi faciliter l'établissement de stratégies de soutien aux proches aidants submergés ${ }^{13}$. Les études ont jusqu'à présent surtout porté sur les besoins en matière de soins des personnes âgées atteintes de démence vivant en établissement de soins ou en établissement de soins de longue durée aux ÉtatsUnis, ainsi que sur l'utilisation des services par ces personnes ${ }^{14-16}$. Les études en population récentes visant les aînés canadiens atteints de démence résidant dans la collectivité sont rares. De plus, il se peut que les travaux antérieurs (issus en grande partie de l'Étude sur la santé et le vieillissement au Canada de 1991 à 2001 ${ }^{17,18}$ ) ne reflètent pas les changements liés à la complexité des besoins en matière de soins ou de services des personnes atteintes de démence et de leurs aidants. Il existe également peu d'études portant sur les personnes atteintes de démence et d'une maladie neurologique concomitante. Il s'agit pourtant d'une sous-population à ne pas négliger, étant donné la hausse probable du stress chez les aidants et de l'utilisation de services de santé découlant de la gravité croissante des symptômes associés à ces maladies neurologiques concomitantes ${ }^{19,20}$.

Pour combler les lacunes touchant les connaissances et les politiques relatives aux soins et à la qualité de vie des aînés canadiens atteints de démence, nous nous sommes fixé comme objectifs d'une part de fournir une synthèse des caractéristiques sociodémographiques, psychosociales et sanitaires d'une vaste cohorte représentative des clients des soins à domicile atteints de démence et de les comparer avec plusieurs autres groupes de clients et, d'autre part, d'étudier les différences entre les caractéristiques observées chez les clients atteints uniquement de démence et celles observées chez les clients atteints de maladies neurologiques concomitantes (par exemple démence et accident vasculaire cérébral [AVC] ou maladie de Parkinson).

\section{Méthodologie}

\section{Conception et échantillon}

Cette étude transversale s'inscrit dans le cadre d'un programme de recherche plus vaste (Innovations en données, observations et applications pour les personnes atteintes de maladies neurologiques, soit ideas $\mathrm{PNC}]^{21}$ ) visant à fournir des estimations de prévalence ainsi que les profils cliniques des personnes atteintes d'au moins l'une des dix principales maladies neurologiques et recevant des services de soins continus.

Notre échantillon comprenait tous les clients des soins à domicile en Ontario âgés de 50 ans ou plus ayant été évalués à l'aide de l'Instrument d'évaluation des résidents - Soins à domicile (RAI-HC) entre janvier 2003 et décembre 2010. Le RAI-HC fournit une évaluation normalisée complète des caractéristiques sociodémographiques du client, de son état physique et cognitif, de ses problèmes de santé et de certains diagnostics, de ses problèmes de comportement, de sa consommation de médicaments et de l'utilisation de services spécifiques. Depuis 2002, tous les clients devant recevoir des soins de longue durée (c.-à-d. plus de 60 jours) à domicile doivent faire l'objet d'une évaluation à l'aide du RAI-HC, et ces données sont enregistrées dans la base de données de l'Association des Centres d'accès aux soins communautaires de l'Ontario (ACASCO).

Nous avons d'abord exclu les évaluations effectuées à l'aide du RAI-HC en milieu de soins de courte durée à des fins de placement $(7,6 \%$ de l'ensemble des évaluations) et nous avons sélectionné l'évaluation la plus récente concernant chaque client ( $\mathrm{n}=520$ 479). Cet échantillon a été réduit à 488374 personnes à la suite de l'imposition de nos limites d'âge (50 ans à 115 ans). Nous avons également exclu les clients évalués avant 2003 (0,02\%) en raison d'incertitudes quant à l'exhaustivité des données recueillies au cours de l'étape initiale de mise en œuvre. L'échantillon final comprenait 488290 clients.

L’Université de Waterloo héberge des copies anonymisées des données dans le cadre d'un contrat de licence conclu entre interRAI et l'Institut canadien d'information sur la santé ${ }^{22}$. Ces fonds de données sont assujettis à des réglements visant à protéger la vie privée, mais ne nécessitent pas le consentement personnel des clients (au-delà de celui obtenu par l'organisme participant au moment de l'évaluation).

Notre étude a reçu l'approbation du Bureau d'éthique de la recherche de l’Université de Waterloo (projet n¹7045).

\section{Mesures}

Des gestionnaires de cas bien formés, habituellement du personnel infirmier ou des travailleurs sociaux, effectuent les vérifications courantes pour le RAI-HC à partir de la meilleure information dont ils disposent (jugement clinique, discussions de cas avec le médecin traitant ou d'autres fournisseurs de soins professionnels et les membres de la famille, examen du dossier médical, etc.). La fiabilité et la validité de l'instrument ont été établies pour diverses populations et milieux ${ }^{23-26}$.

Nous avons utilisé les éléments suivants du RAI-HC : les données sociodémographiques (âge, sexe, état matrimonial, compromis lors de l'achat du traitement requis en raison d'un budget limité), les caractéristiques psychosociales (présence d'un aidant, mode de vie, présence de détresse chez l'aidant), l'état de santé (déficit cognitif et fonctionnel, santé instable, symptômes de dépression et autres symptômes neuropsychiatriques, problèmes de comportement, diagnostic de certaines maladies), les consultations aux urgences et hospitalisations récentes et enfin la consommation de médicaments au cours de la semaine précédente (c.-à-d. 
neuf médicaments ou plus, ou au moins un médicament de certaines catégories [antipsychotique, anxiolytique, antidépresseur, hypnotique, inhibiteur de la cholinestérase ou mémantine]). Les détails concernant les médicaments consommés au cours de la semaine précédente sont consignés manuellement à partir des contenants, vérifiés auprès des clients ou des aidants et transcrits électroniquement.

Nous avons examiné cinq échelles validées issues des éléments du RAI-HC : l'échelle de performance cognitive (CPS, de 0 à 6$)^{27}$, l'échelle hiérarchique d'autoévaluation des activités de la vie quotidienne (AVQ, de 0 à 6) ${ }^{24,28}$, l'échelle de mesure des changements de l'état de santé, des maladies en phase terminale, des signes et des symptômes (CHESS, de 0 à 5$)^{29,30}$, la méthode d'attribution des niveaux de priorité (MAPLe, de 1 à 5$)^{31}$ et l'échelle de mesure de la dépression (DRS, de 0 à 14$)^{32,33}$. Nous avons également examiné une échelle des comportements agressifs (ABS) modifiée ${ }^{34}$ correspondant à la somme des occurrences de quatre types de comportement (violence verbale, violence physique, comportement socialement inapproprié et résistance aux soins) observés au cours des trois jours précédents ainsi qu'une mesure sommaire du degré de difficulté à accomplir quatre activités instrumentales de la vie quotidienne (AIVQ) (préparation des repas, gestion financière, gestion des médicaments et transports). Plus le score sur ces échelles est élevé, plus l'incapacité est importante.

L'échelle CPS illustre l'ampleur du déficit cognitif et a été validée à l'aide du miniexamen de l'état mental ${ }^{35}$. Elle comprend quatre éléments (mémoire à court terme, habiletés cognitives nécessaires à la prise de décisions au quotidien, capacité d'expression et capacité à s'alimenter seul) et ses valeurs vont de 0 (intact) à 6 (déficit très grave) $^{27,35}$. L'échelle CHESS va de 0 (état stable) à 5 (état instable) et associe les symptômes (vomissements, déshydratation, diminution de la consommation d'aliments ou de liquides, perte de poids, essoufflement et œdème) aux éléments permettant de repérer soit un déclin récent (sur le plan des fonctions cognitives et des AVQ), soit la phase terminale de la maladie. Les scores élevés à l'échelle CHESS permettent de prédire la mortalité, le placement en établissement et l'hospitalisation des personnes âgées dans divers milieux de soins ${ }^{29,36,37}$. La méthode MAPLe classe les clients selon cinq niveaux de priorité (de faible à très élevé) en fonction de l'ampleur du déficit cognitif et des incapacités relatives aux AVQ, des problèmes de comportement, des préoccupations liées à l'environnement et du degré d'autonomie. Les scores élevés permettent de prédire le placement en établissement et le stress chez les aidants $^{31}$.

Le RAI-HC comprend une liste de vérification des diagnostics d'affections courantes au sein de la population âgée. Ces affections sont considérées comme présentes si un médecin les a diagnostiquées, si leur traitement ou leur suivi a nécessité des soins professionnels à domicile ou si elles ont été la cause d'une hospitalisation dans les 90 jours précédents. Les maladies neurologiques figurant dans cette liste sont la démence (maladie d'Alzheimer ou autre type de démence), la sclérose en plaques (SP), la maladie de Parkinson ou le parkinsonisme (MP), le traumatisme craniocérébral (TCC, appelé « traumatisme crânien " dans l'instrument) et l'AVC. L'instrument comprend des champs vierges destinés à l'ajout de diagnostics ne figurant pas dans la liste. Six maladies neurologiques ont été codées comme présentes ou absentes à partir de l'examen des diagnostics ajoutés dans ces champs : épilepsie / trouble épileptique, maladie de Huntington, dystrophie musculaire, paralysie cérébrale, traumatisme médullaire et sclérose latérale amyotrophique. Les termes diagnostiques ajoutés ont été définis par consensus par un comité d'examen composé de neurologues, de psychiatres et de gériatres. Les maladies énumérées cidessus (à l'exception de l'AVC) figurent parmi les dix principales maladies neurologiques ciblées par l'Agence de la santé publique du Canada dans le cadre du programme ideas PNC. Nous avons inclus l'AVC dans nos analyses parce qu'il s'agit d'une affection incapacitante que l'on observe couramment chez les personnes âgées.
Les données corroborant l'exactitude des diagnostics consignés dans les instruments RAI ont été publiées ailleurs ${ }^{37-39}$. Wodchis et collab. ${ }^{38}$ ont établi que la sensibilité s'élevait à 0,80 ou plus dans le cas de plusieurs affections observées couramment dans les établissements de soins continus complexes en Ontario (AVC, diabète, cancer, maladie pulmonaire obstructive chronique [MPOC], insuffisance cardiaque, etc.). Des estimations de la sensibilité comparables ont été observées pour la maladie de Parkinson $(0,87)$, la maladie d'Alzheimer $(0,85$, en acceptant que soit coché, dans le RAI, « maladie d'Alzheimer » ou " démence autre que la maladie d'Alzheimer »), la paralysie cérébrale $(0,84)$ et le trouble épileptique $(0,75)$. Les estimations de la sensibilité étaient faibles (inférieures à 0,50 ) pour les autres maladies neurologiques, notamment le traumatisme craniocérébral et la sclérose en plaques.

\section{Analyses}

Nous avons effectué des analyses descriptives de la répartition des caractéristiques sociodémographiques, psychosociales et sanitaires pour les groupes de comparaison suivants : " démence ", " AVC ", " autre maladie neurologique " (présence d'au moins l'une des principales maladies neurologiques : sclérose en plaques, maladie de Parkinson, traumatisme craniocérébral, maladie de Huntington, dystrophie musculaire, paralysie cérébrale, traumatisme médullaire, sclérose latérale amyotrophique, épilepsie) et " témoins sans atteinte cognitive » (clients atteints d'aucune des 11 maladies neurologiques retenues et avec un score de 0 ou 1 sur l'échelle CPS).

Nous avons également effectué des analyses descriptives pour comparer les caractéristiques des personnes atteintes uniquement de démence et de celles atteintes de démence et de certaines autres maladies neurologiques documentées (à savoir " démence et AVC seulement ", " démence et MP seulement ", " démence, MP et AVC seulement » et " démence et TCC seulement »). Ces groupes de comparaison excluaient les clients atteints de l'une des autres maladies neurologiques retenues. 


\section{Résultats}

Clients atteints de démence et clients des groupes "AVC », " autre maladie neurologique » et "témoins sans atteinte cognitive » (tableaux 1A et 1B)

Notre analyse a porté sur 104802 clients $(21,5 \%)$ ayant reçu un diagnostic de démence, 85579 clients $(17,5 \%)$ ayant subi un AVC et 23007 clients $(4,7 \%)$ atteints d'au moins l'une des autres maladies neurologiques principales (20 972 clients [4,3 \%] avaient reçu un diagnostic de démence et un diagnostic d'AVC). Près de la moitié des clients ( $\mathrm{n}=236763 ; 48,5 \%$ ) appartenaient au groupe des témoins sans atteinte cognitive. Nous avons exclu de nos analyses 59089 clients $(12,1 \%)$ présentant un déficit cognitif significatif (score de 2 ou plus à l'échelle CPS) mais n'ayant reçu aucun diagnostic de l'une des principales maladies neurologiques, ainsi que 22 clients pour lesquels il manquait les valeurs sur l'échelle CPS.

Par rapport aux groupes " AVC » et " autre maladie neurologique ", le groupe " démence " comptait un plus grand pourcentage de femmes $(63,7 \%)$ et était composé de personnes plus âgées, l'âge moyen (écart-type) se situant à 83,2 $(7,6)$ ans. Dans l'ensemble des groupes, le pourcentage de femmes mariées était beaucoup moins élevé que celui d'hommes mariés. Relativement peu de clients avaient fait des compromis d'ordre financier, et ceux qui l'avaient fait se retrouvaient davantage dans le groupe de clients atteints d'une autre maladie neurologique que dans celui des clients atteints de démence. Il y avait davantage de clients cohabitant avec leur principal aidant dans les trois groupes de personnes ayant reçu un diagnostic que dans le groupe témoin. Parmi les groupes de clients atteints de démence ou ayant subi un AVC, cet aidant était le plus souvent un enfant, une bru ou un gendre. Chez les clients atteints de démence, on a observé plus fréquemment des conflits interpersonnels, un aidant en détresse, un déficit cognitif modéré ou grave, d'importantes difficultés à accomplir les activités instrumentales de la vie quotidienne (AIVQ) et des difficultés dans les AVQ (tableau 1A).
Les clients atteints de démence étaient plus nombreux que les clients des autres groupes à présenter une certaine instabilité quant à leur état de santé et un degré considérablement plus élevé d'agressivité, d'errance, d'anxiété et d'hallucinations ou de délire (tableau 1B). Ces observations aident à comprendre pourquoi un pourcentage beaucoup plus élevé de clients atteints de démence ont obtenu un score élevé ou très élevé à la MAPLe (figure 1) et ont des aidants en détresse. Dans tous les sous-groupes, une augmentation du pourcentage de clients ayant un aidant en détresse correspondait à une augmentation du score à la MAPLe (figure 2). Toutefois, pour tous les niveaux de la MAPLe, le pourcentage de clients ayant un aidant en détresse était relativement plus élevé dans le groupe des personnes atteintes de démence que dans les groupes de comparaison. Les symptômes de dépression importants sur le plan clinique étaient légèrement plus fréquents chez les clients atteints de démence que chez ceux des groupes de comparaison, bien que la dépression et l'anxiété ne soient pas rares chez les clients atteints d'une autre maladie neurologique ou ayant subi un AVC.

Dans tous les groupes, les affections concomitantes les plus fréquentes étaient les maladies cardiovasculaires, l'arthrite et le diabète. La plupart des diagnostics cliniques étaient moins présents chez les clients atteints de démence ou d'une autre maladie neurologique que chez ceux ayant subi un AVC ou faisant partie du groupe témoin. Les trois groupes de clients ayant reçu un diagnostic de maladie neurologique présentaient une plus faible prévalence de cancer. Les clients atteints d'une autre maladie neurologique étaient les plus nombreux à avoir fait une chute récemment ou à présenter une démarche instable ou des plaies de pression, suivis des clients ayant subi un AVC. Les problèmes de déglutition étaient plus fréquents au sein des trois groupes de clients ayant reçu un diagnostic de maladie neurologique, et notamment au sein du groupe de clients atteints de démence, que dans le groupe de clients sans atteinte cognitive.

Un plus faible pourcentage de clients atteints de démence que de clients des autres groupes avait consulté au moins une fois aux urgences ou subi une hospitalisation au cours des 90 jours précédents ou prenait au moins neuf médicaments. Les clients atteints de démence étaient en revanche plus nombreux que les clients des autres groupes à prendre un antipsychotique ou un neuroleptique. Les clients atteints d'une autre maladie neurologique prenaient plus couramment d'autres psychotropes. La consommation de multiples médicaments $(9$ ou plus) était plus fréquente chez les patients ayant subi un AVC, sans doute en raison du nombre relativement élevé d'affections concomitantes (dont le diabète et les maladies cardiovasculaires). Environ la moitié des clients atteints de démence prenaient un médicament pour le traitement de la démence.

Les clients ayant subi un AVC ou atteints de démence ou d'une autre maladie neurologique étaient plus nombreux que les clients sans atteinte cognitive à avoir reçu des soins par des aidessoignants à domicile $(61 \%$ à $66 \%$ contre $48 \%$ ) et avoir eu recours à des services ménagers à domicile (42 \% contre $31 \%$ ) au cours des sept jours précédents, mais étaient moins nombreux à avoir reçu des soins à domicile par une infirmière autorisée (25 \% à $28 \%$ contre $40 \%$ ). Les clients atteints de démence étaient également moins nombreux que les clients de tous les autres groupes à avoir reçu des services de physiothérapie (7 \% contre $13 \%$ à $15 \%)$ ou d'ergothérapie (8 \% contre $10 \%$ à $16 \%$ ) au cours de la semaine précédente (données non présentées, mais fournies sur demande).

\section{Clients atteints uniquement de démence et clients atteints de démence et d'autres maladies neurologiques (tableaux 2A et 2B)}

La cohorte de clients atteints de démence comprenait 77670 personnes atteintes uniquement de démence (74,1\%), 19061 personnes atteintes de démence et ayant subi un AVC (18,2\%), 4480 personnes atteintes de démence et de la MP (4,3 \%), 1182 personnes atteintes de démence, de la MP et ayant subi un AVC $(1,1 \%)$ et 763 personnes atteintes de démence et ayant subi un TCC $(0,7 \%)$ 
TABLEAU 1A

Caractéristiques sociodémographiques, psychosociales et fonctionnelles des clients des soins à domicile selon leur groupe de diagnostic, Ontario (Canada), 2003-2010

\begin{tabular}{|c|c|c|c|c|}
\hline \multirow[b]{2}{*}{ Caractéristiques } & \multicolumn{4}{|c|}{ Pourcentage (intervalle de confiance à $95 \%)^{\mathrm{a}}$} \\
\hline & $\begin{array}{l}\text { Sans atteinte cognitive }{ }^{b} \\
\quad(n=236763)\end{array}$ & $\begin{array}{l}\text { Autre maladie neurologique }{ }^{c} \\
\qquad(\mathrm{n}=23007)\end{array}$ & $\begin{array}{c}\text { AVC } \\
(n=85579)\end{array}$ & $\begin{array}{c}\text { Démence } \\
(\mathrm{n}=104802)\end{array}$ \\
\hline \multicolumn{5}{|l|}{ Sociodémographiques } \\
\hline Âge moyen (É-T) (ans) & $77,1(11,4)$ & $73,3(11,8)$ & $80,5(9,5)$ & $83,2(7,6)$ \\
\hline 85 ans et plus & $27,8(27,6$ à 28,0$)$ & $17,2(16,7$ à 17,7$)$ & $35,1(34,8$ à 35,4$)$ & $43,6(43,3$ à 43,9$)$ \\
\hline \multicolumn{5}{|l|}{ Sexe } \\
\hline \multicolumn{5}{|l|}{ Marié(e) } \\
\hline Femme & $30,1(29,9$ à 30,3$)$ & $37,8(36,9$ à 38,6$)$ & $27,1(26,7$ à 27,5$)$ & $26,9(26,6$ à 27,2$)$ \\
\hline Homme & $59,9(59,5$ à 60,2$)$ & $61,5(60,6$ à 62,5$)$ & $63,9(63,4$ à 64,4$)$ & $65,5(65,1$ à 66,0$)$ \\
\hline \multicolumn{5}{|l|}{ Veuf(ve) } \\
\hline Femme & 54,3 (54,1 à 54,6) & $39,3(38,5$ à 40,1$)$ & $61,6(61,2$ à 62,1$)$ & $64,1(63,7$ à 64,5$)$ \\
\hline \multicolumn{5}{|l|}{ Cohabite avec le principal aidant } \\
\hline Non & $46,3(46,1$ à 46,5$)$ & 37,2 (36,5 à 37,8) & $40,7(40,4$ à 41,1$)$ & $42,9(42,6$ à 43,2$)$ \\
\hline Oui & $50,3(50,1$ à 50,5$)$ & $59,5(58,9$ à 60,2$)$ & $57,6(57,2$ à 57,9$)$ & $56,0(55,7$ à 56,3$)$ \\
\hline Sans aidant principal & 3,4 (3,3 à 3,5) & $3,3(3,1$ à 3,6) & 1,7 (1,6 à 1,8) & $1,1(1,0$ à 1,1$)$ \\
\hline \multicolumn{5}{|l|}{ Principal aidant } \\
\hline Enfant, gendre ou bru & 46,5 (46,3 à 46,7) & 34,0 (33,3 à 34,6) & 49,9 (49,6 à 50,2) & $53,7(53,4$ à 54,0$)$ \\
\hline Conjoint & $32,2(32,1$ à 32,4$)$ & 42,3 (41,7 à 43,0) & $35,3(35,0$ à 35,6$)$ & $33,7$ (33,5 à 34,0$)$ \\
\hline Autre membre de la famille & $9,9$ (9,8 à 10,0$)$ & $12,5(12,1$ à 13,0$)$ & 7,7 (7,6 à 7,9) & 7,6 (7,4 à 7,7) \\
\hline Ami ou voisin & 7,9 (7,8 à 8,0) & 7,8 (7,5 à 8,2) & 5,4 (5,2 à 5,5) & 3,9 (3,8 à 4,0) \\
\hline Conflits interpersonnels & $10,1(10,0$ à 10,2$)$ & $12,4(12,0$ à 12,8$)$ & $13,1(12,9$ à 13,3$)$ & $17,2(17,0$ à 17,5$)$ \\
\hline Déficit grave (5 ou 6) & - & 2,9 (2,7 à 3,2) & $7,1(6,9$ à 7,3$)$ & $18,7(18,4$ à 18,9$)$ \\
\hline \multicolumn{5}{|l|}{ Score relatif aux AVQ } \\
\hline Indépendance (0) & 78,4 (78,2 à 78,6) & 46,9 (46,3 à 47,5) & 50,2 (49,9 à 50,5) & $37,2$ (36,9 à 37,5$)$ \\
\hline Supervision/limité (1 ou 2) & $15,4(15,2$ à 15,5$)$ & $26,9$ (26,3 à 27,4$)$ & 28,0 (27,7 à 28,3) & $37,9(37,6$ à 38,2$)$ \\
\hline Aide considérable (3 ou 4) & $4,9(4,9$ à 5,0$)$ & $17,9(17,4$ à 18,4$)$ & $15,3(15,0$ à 15,5$)$ & $18,8(18,5$ à 19,0$)$ \\
\hline Dépendance (5 ou 6) & $1,3(1,2$ à 1,3$)$ & $8,3(8,0$ à 8,7$)$ & $6,5(6,4$ à 6,7$)$ & $6,1(6,0$ à 6,3$)$ \\
\hline \multicolumn{5}{|l|}{ Score relatif aux AIVQ $^{d}$} \\
\hline 0 & 15,2 (15,1 à 15,3) & $4,4(4,1$ à 4,6) & 3,8 (3,7 à 4,0) & $0,7(0,6$ à 0,7$)$ \\
\hline 1 ou 2 & 41,1 (40,9 à 41,3) & 23,3 (22,7 à 23,8) & $17,7(17,5$ à 18,0$)$ & 4,4 (4,3 à 4,5) \\
\hline 3 ou 4 & 43,7 (43,5 à 43,9) & 72,4 (71,8 à 72,9) & 78,4 (78,1 à 78,7) & $95,0(94,8$ à 95,1$)$ \\
\hline
\end{tabular}

Abréviations : AIVQ, activité instrumentale de la vie quotidienne; AVC, accident vasculaire cérébral; AVQ, activité de la vie quotidienne; CPS, échelle de performance cognitive; É-T, écart-type; RAI-HC, Instrument d'évaluation des résidents - Soins à domicile.

${ }^{\text {a }}$ Sauf indication contraire.

b Clients n'étant atteints d'aucune des 11 maladies neurologiques retenues et ayant obtenu un score de 0 ou 1 à l'échelle CPS.

c Clients atteints d'au moins l'une des autres maladies neurologiques retenues (à l'exclusion de la démence et des AVC).

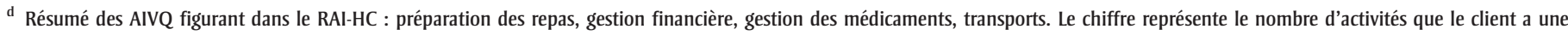
certaine difficulté ou beaucoup de difficulté à réaliser sans aide. 
TABLEAU 1B

Caractéristiques sanitaires des clients des soins à domicile selon le groupe de diagnostic, Ontario (Canada), 2003-2010

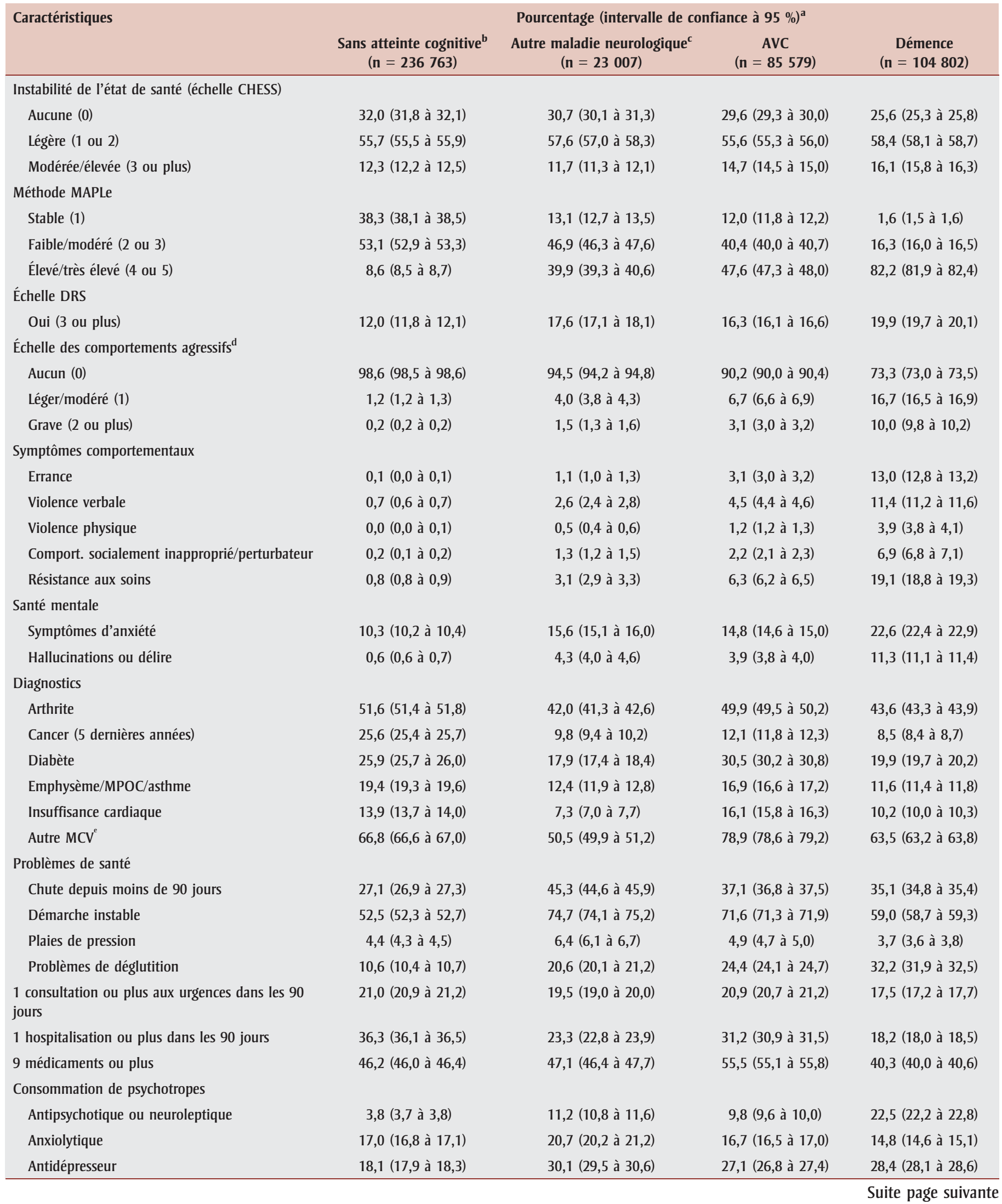


TABLEAU 1B (Suite)

Caractéristiques sanitaires des clients des soins à domicile selon le groupe de diagnostic, Ontario (Canada), 2003-2010

\begin{tabular}{|c|c|c|c|c|}
\hline \multirow[t]{2}{*}{ Caractéristiques } & \multicolumn{4}{|c|}{ Pourcentage (intervalle de confiance à $95 \%)^{a}$} \\
\hline & $\begin{array}{l}\text { Sans atteinte cognitive }{ }^{b} \\
\quad(n=236763)\end{array}$ & $\begin{array}{l}\text { Autre maladie neurologique }{ }^{c} \\
\qquad(\mathrm{n}=23007)\end{array}$ & $\begin{array}{c}\text { AVC } \\
(n=85579)\end{array}$ & $\begin{array}{c}\text { Démence } \\
\text { (n = } 104802)\end{array}$ \\
\hline Hypnotique & $14,2(14,1$ à 14,4$)$ & $15,6(15,1$ à 16,1$)$ & $14,3(14,1$ à 14,5$)$ & $11,8(11,6$ à 12,0$)$ \\
\hline Tout médicament pour le traitement de la démence & $0,9(0,9$ à 1,0$)$ & $5,0(4,7$ à 5,3$)$ & $13,0(12,8$ à 13,2$)$ & $49,3(49,0$ à 49,6$)$ \\
\hline
\end{tabular}

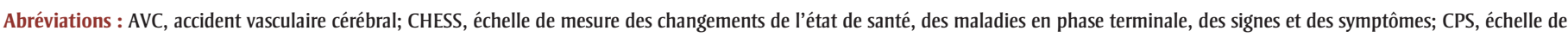

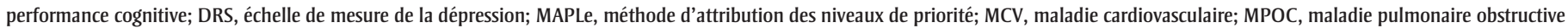
chronique; RAI-HC, Instrument d'évaluation des résidents - Soins à domicile.

a Sauf indication contraire.

b Clients n'étant atteints d'aucune des 11 maladies neurologiques retenues et ayant obtenu un score de 0 ou 1 à l'échelle CPS.

c Clients atteints d'au moins une des autres maladies neurologiques retenues (à l'exclusion de la démence et des AVC).

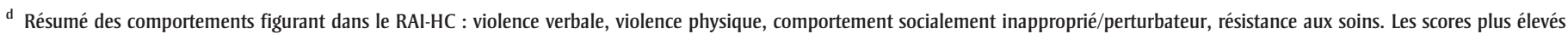
correspondent à un nombre et à une fréquence plus élevés de problèmes de comportement.

e Comprend les maladies cardiovasculaires suivantes figurant dans le RAI-HC : coronaropathie, hypertension, pouls irrégulièrement irrégulier, maladie vasculaire périphérique.

(tableau 2A). On comptait 1646 clients (1,6\%) atteints de démence et d'autres maladies parmi les maladies neurologiques retenues, mais, ces combinaisons étant rares, elles ne sont pas présentées ici.

Les clients atteints de démence et de la MP (avec ou sans AVC) et les clients atteints de démence et ayant subi un TCC étaient généralement plus jeunes et plus souvent de sexe masculin que les clients des deux autres groupes. Cette répartition quant à l'âge et au sexe explique probablement le pourcentage relativement plus élevé de clients mariés (cohabitant avec un conjoint aidant) dans les groupes de clients atteints de démence et de la MP (avec ou sans AVC). Les clients atteints de démence et ayant subi un TCC étaient plus nom-

FIGURE 1

Répartition des niveaux de la MAPLe selon le groupe de diagnostic

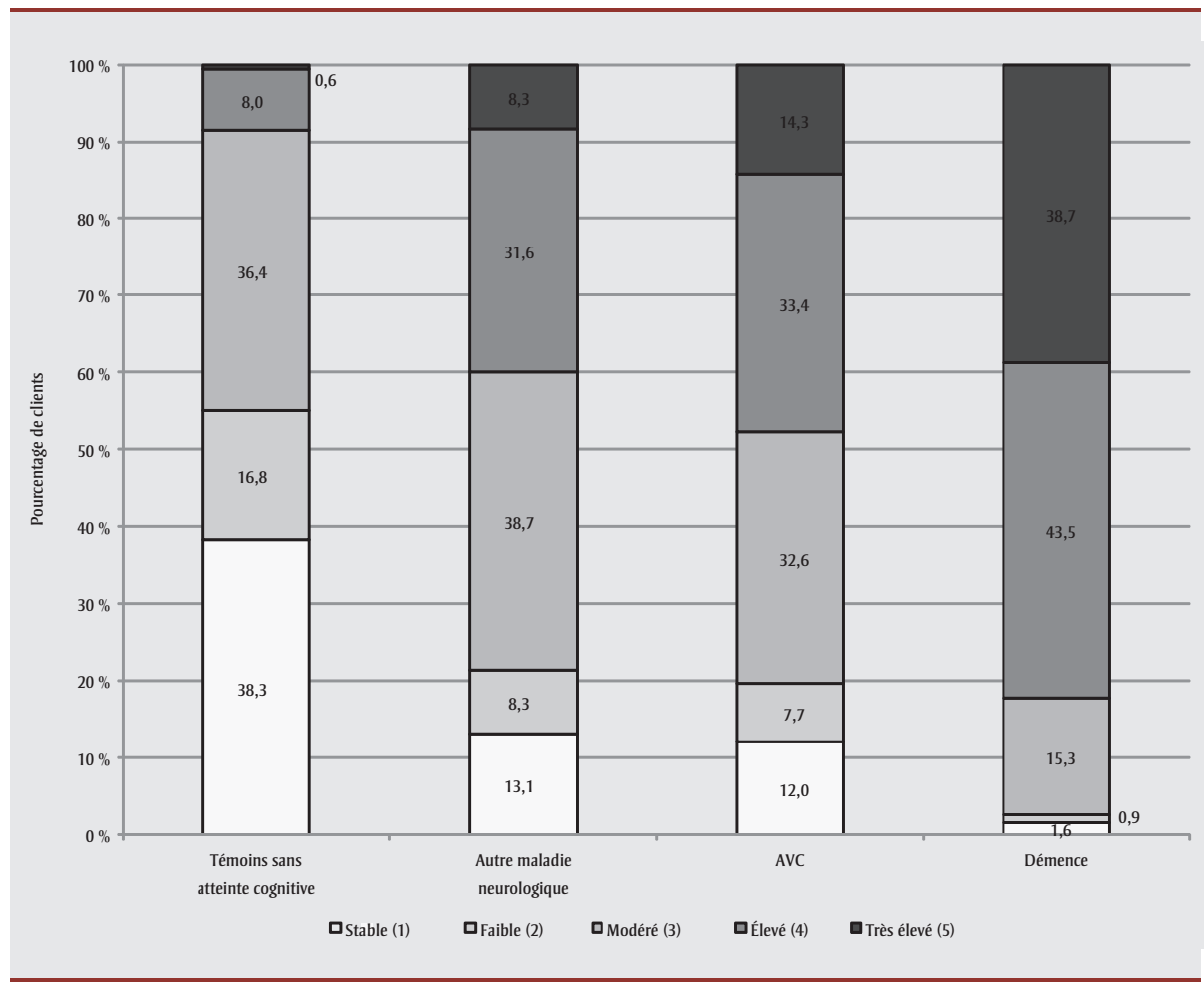

Abréviations : AVC, accident vasculaire cérébral; MAPLe, méthode d'attribution des niveaux de priorité.

breux que ceux des autres groupes à avoir des conflits interpersonnels et, à l'instar des clients atteints de la MP (avec ou sans AVC), ils étaient plus nombreux à avoir un aidant en détresse. Les clients atteints de démence, de la MP et ayant subi un AVC présentaient plus souvent que les clients des autres groupes un déficit cognitif plus important et une plus grande dépendance dans l'exécution des AVQ.

Un état de santé modérément instable ou très instable était observé un peu plus fréquemment chez les clients ayant subi un AVC (avec ou sans MP) ou un TCC (tableau 2B). Tous les groupes ont obtenu des pourcentages similaires aux niveaux élevé et très élevé de la MAPLe et présentaient des symptômes de dépression importants sur le plan clinique. Les comportements agressifs étaient moins fréquents chez les clients atteints de démence et de la MP (avec ou sans AVC) et plus fréquents chez ceux atteints de démence et ayant subi un TCC. Les symptômes d'anxiété étaient légèrement plus courants chez les clients atteints de démence et ayant subi un TCC, tandis que les hallucinations ou le délire étaient davantage observés chez les clients atteints de démence et de la MP.

En règle générale, les clients atteints de démence et ayant subi un AVC étaient plus nombreux à être également atteints de diverses affections concomitantes (dont l'arthrite, le diabète et diverses maladies cardiovasculaires) que les clients atteints 
FIGURE 2

Pourcentage de clients ayant un aidant en détresse selon le score à la MAPLe et le groupe de diagnostic

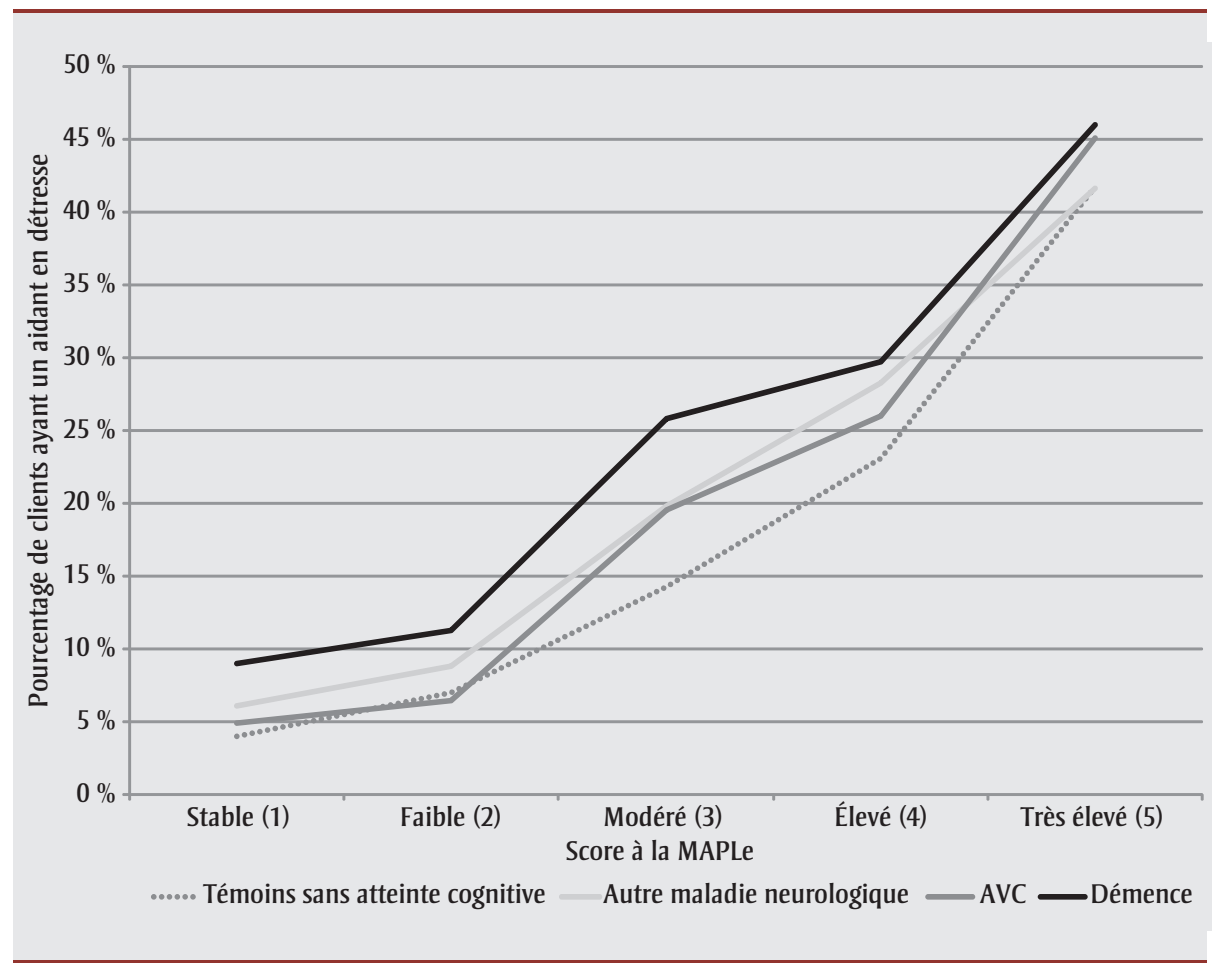

Abréviations : AVC, accident vasculaire cérébral; MAPLe, méthode d'attribution des niveaux de priorité.

de démence et de la MP seulement. Par contre, on signalait plus souvent chez ces derniers une démarche instable, des plaies de pression et une chute récente. Dans l'ensemble, par rapport aux clients atteints uniquement de démence, les clients des quatre groupes atteints d'une maladie neurologique concomitante étaient plus nombreux à avoir fait une chute récemment et à présenter une démarche instable et des problèmes de déglutition (ces derniers étaient particulièrement courants chez les clients atteints à la fois de démence, de la MP et ayant subi un $A V C)$. Les clients des quatre groupes atteints d'une maladie neurologique concomitante étaient plus nombreux que les clients atteints uniquement de démence à avoir récemment consulté aux urgences ou à avoir été hospitalisés. Les clients atteints de démence et ayant subi un AVC (avec ou sans MP) ou un TCC avaient particulièrement fréquemment été hospitalisés récemment. La consommation d'au moins neuf médicaments était moins fréquente chez les clients des groupes " démence uniquement" et "démence et TCC " que chez ceux des groupes "démence et AVC », « démence et MP » et plus particulièrement « démence et AVC et MP ».

Les clients atteints de démence et de la MP (avec ou sans AVC) étaient généralement plus nombreux que ceux des autres groupes à consommer des antipsychotiques ou des neuroleptiques et des antidépresseurs. Les clients des groupes " démence et MP » (sans AVC) et " démence uniquement " étaient plus nombreux que ceux des autres groupes à consommer des inhibiteurs de la cholinestérase ou de la mémantine, tandis que ceux des groupes " démence et TCC » et " démence et AVC » (sans MP) étaient les moins nombreux à en consommer.

\section{Analyse}

Les constatations issues de cette étude en population des clients des soins à domicile en Ontario font ressortir les besoins considérables, sur les plans tant psychosocial, fonctionnel que de la santé mentale, des personnes atteintes de démence qui vivent dans la collectivité. Nos travaux permettent d'étoffer les recherches antérieures en fournissant un profil récent et complet des principaux domaines liés aux soins, à la qualité de vie et aux résultats en matière de santé de cette population, dont les effectifs sont en croissance. De plus, nous fournissons des estimations de la prévalence de certaines maladies neurologiques concomitantes courantes et de la complexité des besoins qui en découlent en matière de planification de la santé et des soins.

Clients atteints de démence et clients des groupes «AVC», « autre maladie neurologique » et «témoins sans atteinte cognitive »

Environ $22 \%$ des clients recevant des soins de longue durée à domicile en Ontario (n = 104 802) avaient reçu un diagnostic de démence. L'un des profils fréquents était celui d'une veuve âgée (plus de 75 ans) dont l'enfant (ou le gendre ou la bru) agissait à titre d'aidant principal. Cependant, chez environ le tiers des clients atteints de démence, le principal aidant était le conjoint, et ce dernier, le plus souvent du même âge ou plus vieux, était souvent confronté à court terme à des problèmes liés à sa propre santé et à son propre bien-être social. Environ $43 \%$ des clients atteints de démence (et $50 \%$ des personnes ayant un déficit cognitif, mais sans diagnostic) ne cohabitaient pas avec leur principal aidant, or l'absence d'une personne proche ou bien informée pouvant s'enquérir de leurs besoins et les communiquer rapidement peut constituer un risque accru de fragmentation ou de prestation sous-optimale des soins aux malades et conduire à une progression plus rapide de leur maladie ${ }^{40,41}$.

Nous avons vu que près de la moitié des clients atteints de démence présentaient un déficit cognitif modéré ou grave (score de 3 ou plus sur l'échelle CPS) et que presque tous avaient une certaine difficulté ou beaucoup de difficulté à exécuter de nombreuses AIVQ. Comme les coûts des soins, professionnels et autres, augmentent avec la gravité de la démence ${ }^{3,6,42}$, cette observation a une grande incidence sur les proches aidants, les fournisseurs de soins de santé et les décideurs. Compte tenu du degré de gravité de leur déficit cognitif, les clients atteints de démence présentaient beaucoup plus souvent que les clients des 
TABLEAU 2A

Caractéristiques sociodémographiques, psychosociales et fonctionnelles des clients des soins à domicile atteints de démence (selon la maladie neurologique concomitante), Ontario (Canada), 2003-2010

\begin{tabular}{|c|c|c|c|c|c|}
\hline \multirow[t]{2}{*}{ Caractéristiques } & \multicolumn{5}{|c|}{ Pourcentage (intervalle de confiance à $95 \%)^{\mathrm{a}}$} \\
\hline & $\begin{array}{l}\text { Démence uniquement }{ }^{\mathbf{b}} \\
\qquad(\mathrm{n}=77670)\end{array}$ & $\begin{array}{l}\text { Démence et } \text { AVC }^{c} \\
\qquad(n=19061)\end{array}$ & $\begin{array}{l}\text { Démence et MPc } \\
\qquad(\mathrm{n}=4480)\end{array}$ & $\begin{array}{l}\text { Démence, MP et } \mathrm{AVC}^{\mathrm{d}} \\
(\mathrm{n}=1 \mathrm{182})\end{array}$ & $\begin{array}{l}\text { Démence et TCC } \\
\qquad(n=763)\end{array}$ \\
\hline Âge moyen (É-T), années & $83,5(7,5)$ & $83,3(7,3)$ & $80,6(6,9)$ & $81,7(6,6)$ & $80,1(9,6)$ \\
\hline 85 ans et plus & 45,4 (45,1 à 45,8) & 43,2 (42,5 à 43,9) & $26,5(25,2$ à 27,8$)$ & $30,1(27,5$ à 32,7$)$ & $35,0(31,6$ à 38,4$)$ \\
\hline \multicolumn{6}{|l|}{ Marié(e) } \\
\hline Homme & $63,0(62,4$ à 63,6$)$ & $68,6(67,6$ à 69,6) & 77,6 (76,0 à 79,2) & $78,0(75,0$ à 81,1$)$ & 61,9 (56,9 à 66,8) \\
\hline Femme & $26,4$ (26,0 à 26,7$)$ & $26,6(25,8$ à 27,5$)$ & $38,7(36,5$ à 40,9$)$ & $30,6(26,4$ à 34,8$)$ & $29,8(25,3$ à 34,3$)$ \\
\hline Compromis d'ordre financier & $0,9(0,8$ à 1,0$)$ & $1,3(1,1$ à 1,4$)$ & $1,3(0,9$ à 1,6$)$ & $1,1(0,5$ à 1,7$)$ & $2,1(1,1$ à 3,1$)$ \\
\hline Oui & $53,4(53,0$ à 53,7$)$ & $62,1(61,4$ à 62,8) & $69,8(68,4$ à 71,1$)$ & $74,2(71,7$ à 76,7$)$ & $54,7(51,2$ à 58,3$)$ \\
\hline Sans aidant principal & $1,1(1,1$ à 1,2$)$ & $0,8(0,7$ à 1,0$)$ & $0,7(0,5$ à 1,0$)$ & $0,3(0,0$ à 0,7$)$ & $1,7(0,8$ à 2,6$)$ \\
\hline \multicolumn{6}{|l|}{ Principal aidant } \\
\hline Enfant, gendre ou bru & $55,4(55,1$ à 55,8$)$ & 52,4 (51,7 à 53,2) & 38,0 (36,5 à 39,4) & $42,6(39,8$ à 45,5) & $46,8(43,2$ à 50,3$)$ \\
\hline Conjoint & $31,2(30,8$ à 31,5$)$ & $37,6(36,9$ à 38,3$)$ & $53,9(52,4$ à 55,3$)$ & $51,4(48,6$ à 54,3$)$ & 37,4 (33,9 à 40,8) \\
\hline Autre membre de la famille & $8,1(7,9$ à 8,3) & 6,0 (5,6 à 6,3) & 4,8 (4,2 à 5,4) & $3,7(2,6$ à 4,8) & $9,6$ (7,5 à 11,7$)$ \\
\hline Ami/voisin & $4,1(4,0$ à 4,3$)$ & $3,2(2,9$ à 3,4$)$ & $2,6(2,1$ à 3,1$)$ & $1,9(1,1$ à 2,6$)$ & $4,5(3,0$ à 5,9$)$ \\
\hline Conflits interpersonnels & $17,4(17,2$ à 17,7$)$ & $17,1(16,6$ à 17,6) & $14,5(13,5$ à 15,5$)$ & $14,0(12,0$ à 15,9$)$ & $21,7(18,7$ à 24,6$)$ \\
\hline Aidant en détresse & 34,4 (34,1 à 34,8) & 35,2 (34,5 à 35,8) & $39,3$ (37,9 à 40,7$)$ & 39,5 (36,7 à 42,3) & 40,4 (36,9 à 43,9) \\
\hline \multicolumn{6}{|l|}{ Fonctionnelles } \\
\hline Indépendance (0) & 39,9 (39,6 à 40,3) & 31,8 (31,1 à 32,4) & $21,0(19,8$ à 22,2$)$ & $17,5$ (15,3 à 19,7$)$ & $36,8(33,4$ à 40,3$)$ \\
\hline Supervision/limité (1 ou 2) & $38,2(37,9$ à 38,6) & $37,0(36,3$ à 37,7$)$ & $37,2(35,7$ à 38,6$)$ & $34,1(31,4$ à 36,8$)$ & $37,6(34,2$ à 41,1$)$ \\
\hline Aide considérable (3 ou 4) & $17,1(16,8$ à 17,3$)$ & 21,8 (21,2 à 22,4) & $30,8(29,5$ à 32,2$)$ & $30,2(27,6$ à 32,8) & $20,1(17,2$ à 22,9$)$ \\
\hline Dépendance (5 ou 6) & 4,8 (4,6 à 4,9) & 9,4 (9,0 à 9,8) & $11,1(10,1$ à 12,0$)$ & $18,2(16,0$ à 20,4$)$ & 5,5 (3,9 à 7,1) \\
\hline \multicolumn{6}{|l|}{ Score relatif aux AIVQ $^{\mathrm{e}}$} \\
\hline 0 & $0,8$ (0,7 à 0,8$)$ & $0,4(0,3$ à 0,5$)$ & $0,2(0,1$ à 0,4$)$ & $0,3(0,0$ à 0,5$)$ & 0,7 (0,1 à 1,2) \\
\hline 1 ou 2 & 4,8 (4,6 à 4,9) & 3,3 (3,1 à 3,6) & $2,5(2,1$ à 3,0) & $1,4(0,8$ à 2,1$)$ & 5,5 (3,9 à 7,1) \\
\hline 3 ou 4 & $94,5(94,3$ à 94,6$)$ & $96,2(96,0$ à 96,5) & $97,2(96,8$ à 97,7$)$ & $98,3(97,6$ à 99,0) & $93,8(92,1$ à 95,5$)$ \\
\hline
\end{tabular}

Abréviations : AIVQ, activité instrumentale de la vie quotidienne; AVC, accident vasculaire cérébral; AVQ, activité de la vie quotidienne; CPS, échelle de performance cognitive; É-T, écart-type; MP, maladie de Parkinson/parkinsonisme; TCC, traumatisme craniocérébral.

a Sauf indication contraire.

b À l'exclusion des dix autres maladies neurologiques retenues.

c $\grave{A}$ l'exclusion des neuf autres maladies neurologiques retenues.

d À l'exclusion des huit autres maladies neurologiques retenues.

e Résumé des AIVQ figurant dans le RAI-HC : préparation des repas, gestion financière, gestion des médicaments, transports. Le chiffre représente le nombre d’activités que le client a une certaine difficulté ou beaucoup de difficulté à réaliser sans aide. 
TABLEAU 2B

Caractéristiques sanitaires des clients des soins à domicile atteints de démence (selon la maladie neurologique concomitante), Ontario (Canada), 2003-2010

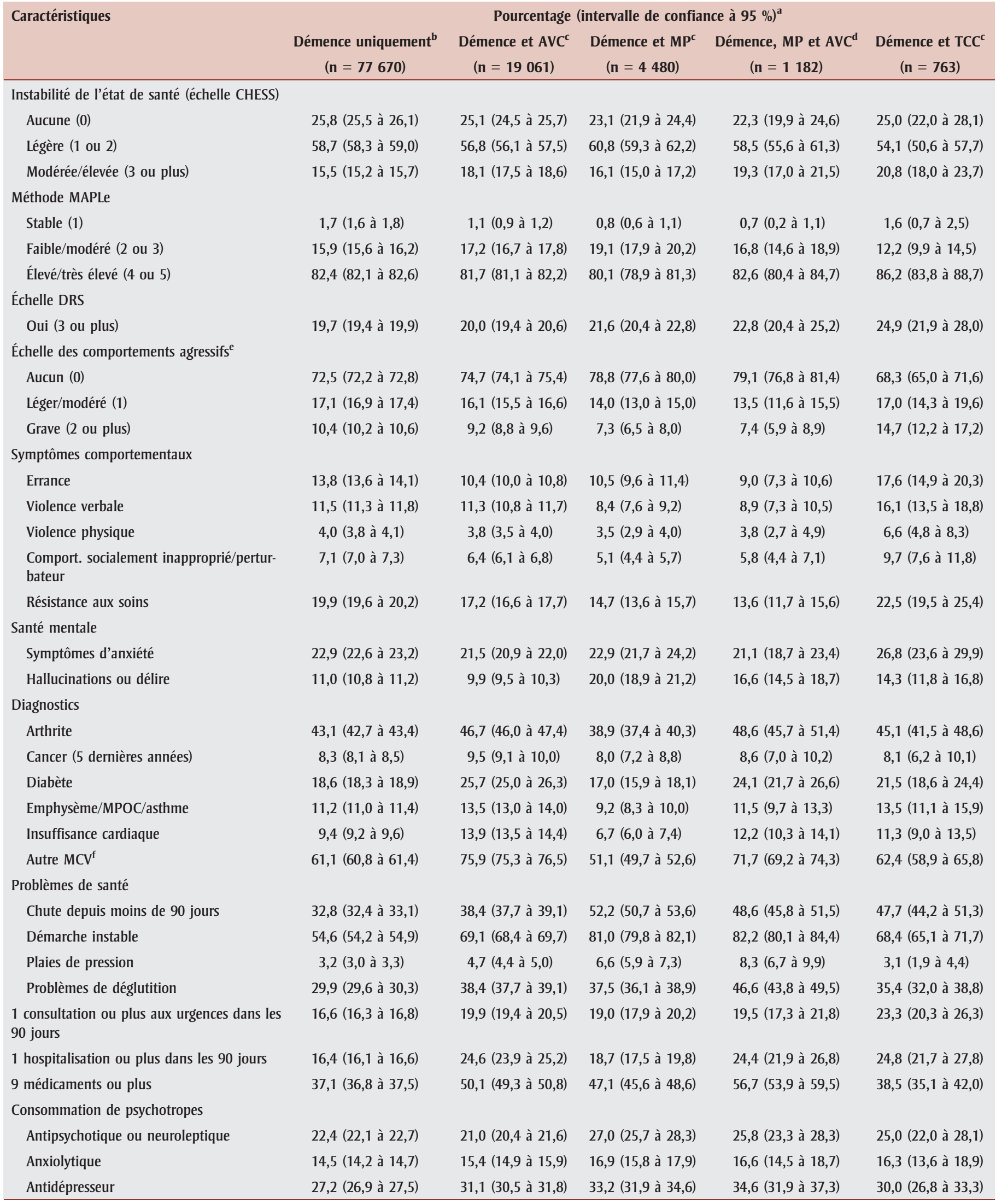


TABLEAU 2B (Suite)

Caractéristiques sanitaires des clients des soins à domicile atteints de démence (selon la maladie neurologique concomitante), Ontario (Canada), 2003-2010

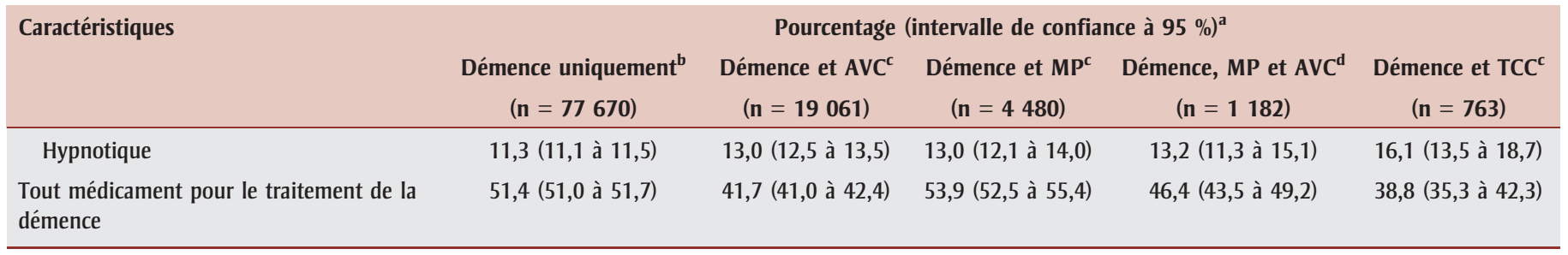

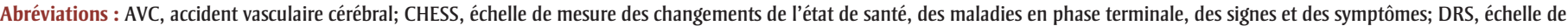

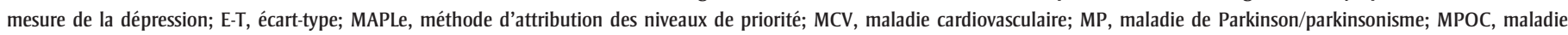
pulmonaire obstructive chronique; RAI-HC, Instrument d'évaluation des résidents - Soins à domicile; TCC, traumatisme craniocérébral.

a Sauf indication contraire.

b À l'exclusion des dix autres maladies neurologiques retenues.

c À l'exclusion des neuf autres maladies neurologiques retenues.

d À l'exclusion des huit autres maladies neurologiques retenues.

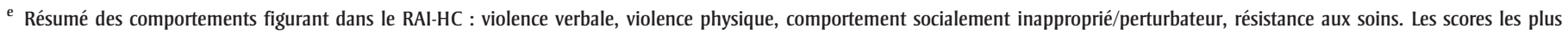
élevés correspondent à un nombre et à une fréquence plus élevés de problèmes de comportement.

f Comprend les maladies cardiovasculaires suivantes figurant dans le RAI-HC : coronaropathie, hypertension, pouls irrégulièrement irrégulier, maladie vasculaire périphérique.

autres groupes de l'agressivité, de l'anxiété, de l'errance et des hallucinations ou du délire. Ils étaient aussi plus nombreux à manifester des symptômes de dépression importants sur le plan clinique. Dans une étude portant sur des personnes de 55 ans ou plus ayant participé à l'Enquête sur la santé dans les collectivités canadiennes de 2005, Nabalamba et Patten ${ }^{43}$ ont également observé des pourcentages plus élevés de troubles de l'humeur $(19,5 \%)$ et de troubles anxieux (16,3\%) chez les personnes atteintes de démence. L'évidente juxtaposition de problèmes cognitifs, comportementaux et psychiatriques chez les clients atteints de démence aide à expliquer le pourcentage plus élevé d'aidants en détresse $^{17}$ (environ $35 \%$ des proches aidants dans notre étude), le risque accru de placement en établissement ${ }^{17,44}$ et le coût plus élevé des soins ${ }^{42,45}$. Plus précisément, 82 \% des clients atteints de démence ont obtenu des scores élevés ou très élevés à la MAPLe, signe d'un risque imminent de transition vers un niveau de soins plus élevé.

Les clients atteints de démence (et ceux atteints d'autres maladies neurologiques) étaient moins souvent atteints de diverses maladies chroniques (maladies cardiovasculaires, arthrite, diabète, MPOC et cancer, notamment) et étaient moins nombreux à avoir eu recours à des services de santé récemment (comme une consultation aux urgences, une hospitalisation au cours des trois derniers mois ou la consommation de neuf médicaments ou plus). Bien que des travaux antérieurs aient révélé que les personnes atteintes de démence (en particulier celles atteintes de la maladie d'Alzheimer) étaient en relativement meilleure santé ${ }^{46,47}$, les études récentes sont moins univoques $^{45,48}$. La seule exception est une prévalence systématiquement moins élevée de cancer chez les personnes atteintes de démence et d'autres maladies neurologiques ${ }^{49}$. Ces incohérences découlent probablement de différences dans la conception et l'échantillonnage des études (entre autres, à propos des caractéristiques sociodémographiques, du degré de gravité et des sous-types de démence examinés) ainsi que dans les mesures diagnostiques et les mesures cliniques de la santé utilisées. Plusieurs études ont signalé des taux plus élevés d'affections concomitantes, de consommation de médicaments et d'utilisation des services de santé chez les personnes atteintes de démence vasculaire (par rapport à celles atteintes de la maladie d'Alzheimer $^{46,48}$. Nos constatations vont dans le même sens en ce qui concerne les clients atteints de démence qui ont subi un AVC (tableau 2B). La prévalence moins élevée de certaines maladies peut être attribuable à une moins bonne détection et au sous-diagnostic chez les personnes atteintes d'un trouble démentiel ${ }^{48}$. Parmi les facteurs qui pourraient sous-tendre cette reconnaissance plus faible, on compte la présentation atypique de certaines maladies, la sous-déclaration des symptômes chez les patients atteins de démence ainsi que le stigmate associé au diagnostic de démence. D’autres études sont nécessaires pour examiner cette possibilité ainsi que d'éventuelles stratégies visant à améliorer la détection de maladies concomitantes chez les patients atteints de démence ${ }^{50}$. Il convient également de noter que les clients atteints de démence étaient beaucoup plus nombreux à connaître des problèmes de déglutition et à consommer des antipsychotiques ou des neuroleptiques, deux facteurs de risque de déclin et d'hospitalisation $^{45,51}$.

\section{Clients atteints uniquement de démence et clients atteints de démence et d'autres maladies neurologiques}

Environ un client sur quatre atteint de démence présentait une maladie neurologique concomitante (parmi celles que nous avons ciblées). Il s'agissait le plus souvent, dans l'ordre, de l'AVC, de la MP et du TCC. D'autres chercheurs ont établi que la démence est relativement fréquente chez les personnes atteintes de la MP ou ayant subi un $\mathrm{AVC}^{20,52}$. Les clients chez qui les 
trois maladies (démence, MP et AVC) avaient été diagnostiquées présentaient les déficits cognitifs les plus graves et les incapacités les plus importantes dans les AVQ. Par rapport aux clients atteints uniquement de démence, ceux des quatre groupes atteints d'une maladie neurologique concomitante étaient plus nombreux à avoir fait une chute récemment, à avoir une démarche instable, des problèmes de déglutition (ces derniers étant présents chez près de $50 \%$ des clients à la fois atteints de démence et de la MP et ayant subi un AVC) et à avoir eu recours à des services de santé récemment.

Les clients atteints de démence et de l'une ou l'autre des maladies neurologiques concomitantes retenues présentaient également des profils sociodémographiques et sanitaires bien particuliers. Les clients atteints de démence et de la MP étaient plus jeunes et ce groupe comptait davantage d'hommes, avec comme conséquence une cohabitation plus fréquente avec un conjoint aidant. On a signalé un aidant en détresse chez environ $40 \%$ des clients des groupes « démence et MP » et " démence et TCC » (par rapport à environ $35 \%$ chez les clients des autres groupes). Cependant, les facteurs sous-jacents pouvant alourdir le fardeau des aidants n'étaient pas les mêmes dans ces deux groupes. Par exemple, les clients atteints de démence et ayant subi un TCC étaient plus nombreux que ceux des autres groupes à vivre des conflits interpersonnels et à présenter des comportements agressifs, de l'errance et une aggravation récente des troubles de l'humeur ou du comportement, alors que, à l'inverse, les clients atteints de démence et de la MP présentaient moins de problèmes de comportement ou de conflits mais davantage d'hallucinations ou de délire.

On a constaté une plus grande instabilité de l'état de santé (c.-à-d. des scores plus élevés à l'échelle CHESS et une utilisation récente des services hospitaliers) chez les clients atteints de démence et ayant subi un AVC ou un TCC. De plus, les clients du groupe "démence et AVC » étaient plus nombreux à être atteints d'une maladie concomitante courante (maladie cardiovasculaire, diabète ou arthrite) et à consommer au moins neuf médicaments.
Même s'ils étaient moins souvent atteints de ces maladies concomitantes, les clients du groupe " démence et MP » étaient plus nombreux à avoir fait une chute récemment et à présenter une démarche instable et des plaies de pression. Les différences observées chez les clients atteints de démence et d'une maladie neurologique concomitante quant à la consommation d'inhibiteurs de la cholinestérase ou de mémantine étaient étonnantes et ont fait l'objet d'un examen plus approfondi dans une autre publication ${ }^{53}$.

Les besoins en matière de soins propres à certains sous-groupes de clients atteints de démence illustrent l'importance d'offrir des soins à domicile personnalisés et coordonnés $^{13}$. Par exemple, de nouvelles ressources éducatives et des stratégies de gestion du comportement pourraient s'avérer prioritaires pour les clients atteints de démence et ayant subi un TCC (et pour leurs aidants), tandis que les clients atteints de démence et de la MP pourraient avoir davantage besoin de stratégies de prévention des chutes et de services de réadaptation, et que les clients atteints de démence et ayant subi un AVC tireraient bénéfice d'une meilleure gestion des maladies chroniques.

Les principaux points forts de notre étude sont, d'une part, l'examen d'un vaste échantillon représentatif de la population de clients âgés recevant des soins à domicile (ce qui permet des estimations plus précises, une stratification selon le sous-groupe de diagnostic et une généralisabilité) et, d'autre part, la nature exhaustive et intégrale des données d'évaluation obtenues à l'aide du RAI-HC. Toutefois, on doit tenir compte de certaines limites. Malgré les éléments probants à l'appui de la validité des données sur les diagnostics obtenues à l'aide du RAI-HC (notamment la démence) ${ }^{37-39}$, d'autres travaux de validation s'imposent. De plus, les données sur les diagnostics et sur les fonctions cognitives obtenues à l'aide du RAI-HC ne permettent pas de différencier les sous-types de démence (pourtant un important prédicteur des besoins en matière de soins et d'utilisation des services). Environ 12,1 \% des clients (sans diagnostic de maladie neurologique indiqué) présentaient un déficit cognitif modéré ou grave et certains d'entre eux (en particulier ceux ayant obtenu un score de 4 ou plus à l'échelle CPS) étaient probablement atteints d'un trouble démentiel. De possibles erreurs de classification des diagnostics pourraient avoir réduit notre capacité de détecter des différences pertinentes entre les caractéristiques des clients de certains de nos groupes de comparaison. La nature transversale de nos données et l'absence de données prospectives sur l'utilisation réelle du système de santé et des soins à domicile limitent également notre capacité de nous prononcer sur les différents fardeaux et besoins non comblés, en matière de soins, qui découlent de la présence, chez les clients atteints de démence, des maladies neurologiques concomitantes retenues.

\section{Conclusion}

Nos résultats indiquent que les clients atteints de démence vivant dans la collectivité pourraient, dans une proportion importante, atteindre bientôt le seuil critique quant à leur capacité de continuer à vivre chez eux. Ces données viennent renforcer l'argument selon lequel les programmes communautaires et les programmes de soutien pour les aidants devraient être assouplis et améliorés afin d'assurer la continuité du bienêtre des clients et de leurs aidants et le maintien de la qualité des soins ${ }^{13,54}$. Ce qui est essentiel pour tous les clients atteints de démence (en raison du fardeau que représentent les troubles de l'humeur et les troubles anxieux) est la présence de services psychiatriques et de services de santé mentale améliorés, coordonnés et intégrés (ce qui inclut la prise en charge des cas graves) ${ }^{43}$. Les fournisseurs de soins (notamment les gestionnaires de cas, les médecins de première ligne et les proches aidants) sont susceptibles de rencontrer de nombreux obstacles structurels lorsqu'ils tentent d'avoir accès à des spécialistes et à des services appropriés en matière de santé mentale ${ }^{55}$. Ces obstacles accroissent le risque, pour les aînés atteints de démence qui résident dans la collectivité, de recevoir des traitements tardifs ou inadéquats, et avec de moins bons résultats. D’autres travaux s'imposent 
pour préciser l'ampleur et les conséquences des besoins non comblés découlant de la présence, chez les clients atteints de démence, de maladies neurologiques et de problèmes de santé mentale concomitants.

\section{Remerciements}

Cette étude relève de l'Étude nationale de la santé des populations relative aux maladies neurologiques. Nous aimerions remercier les Organismes caritatifs neurologiques du Canada et l'Agence de la santé publique du Canada pour leur contribution au succès de cette initiative.

L'étude a été financée par l'Agence de la santé publique du Canada. Les opinions exprimées dans cet article sont celles des auteurs et des chercheurs et ne reflètent cependant pas nécessairement la position de l'Agence de la santé publique du Canada.

D. B. Hogan est titulaire de la Chaire en gériatrie de la Brenda Strafford Foundation. N. Jetté est titulaire du Population Health Investigator Award d'Alberta Innovates Health Solutions et d'une chaire de recherche du Canada (niveau 2) en recherche sur les services de santé neurologique. S. E. Bronskill reçoit une bourse de nouveau chercheur dans le domaine du vieillissement des Instituts de recherche en santé du Canada. J. P. Hirdes est titulaire de la Chaire de recherche et d'échange des connaissances en soins à domicile de l'Ontario financée par le ministère de la Santé et des Soins de longue durée de l'Ontario.

\section{Conflit d'intérêts}

Aucun.

\section{Références}

1. World Health Organization, Alzheimer's Disease International. Dementia: a public health priority. Geneva (CH) : World Health Organization; 2012.

2. Smetanin P, Kobak P, Briante C, Stiff D, Sherman G, Ahmad S. Rising tide: the impact of dementia in Canada 2008 to 2038. Toronto (Ont.) : RiskAnalytica; 2009.
3. Jakobsen M, Poulsen PB, Reiche T, Nissen NP, Gundgaard J. Costs of informal care for people suffering from dementia: evidence from a Danish survey. Dement Geriatr Cogn Dis Extra. 2011;1(1):418-28.

4. Hill J, Fillit H, Thomas SK, Chang S. Functional impairment, healthcare costs and the prevalence of institutionalisation in patients with Alzheimer's disease and other dementias. Pharmacoeconomics. 2006;24(3):265-80.

5. Wimo A, Winblad B, Jönsson L. The worldwide societal costs of dementia: estimates for 2009. Alzheimers Dement. 2010;6(2):98-103.

6. Hux MJ, O’Brien BJ, Iskedjian M, Goeree R, Gagnon M, Gauthier S. Relation between severity of Alzheimer's disease and costs of caring. CMAJ. 1998;159(5):457-65.

7. Hirdes JP, Mitchell L, Maxwell CJ, White N. Beyond the 'iron lungs of gerontology': using evidence to shape the future of nursing homes in Canada. Can J Aging. 2011;30(3):371-90.

8. Agüero-Torres $\mathrm{H}$, von Strauss E, Viitanen M, Winblad B, Fratiglioni L. Institutionalization in the elderly: the role of chronic diseases and dementia. Cross-sectional and longitudinal data from a population-based study. J Clin Epidemiol. 2001;54(8):795801.

9. Williams AP, Challis D, Deber R, et al. Balancing institutional and communitybased care: why some older persons can age successfully at home while others require residential long-term care. Healthc Q. 2009;12(2):95-105.

10. Canadian Home Care Association (CHCA). Portraits of home care in Canada. Mississauga (Ont.) : CHCA; 2008.

11. Institut canadien d'information sur la santé (ICIS). Statistiques éclair du Système d'information sur les services à domicile, 2010-2011. Ottawa (Ont.) : ICIS; 2011.

12. Institut canadien d'information sur la santé. Les soins aux personnes âgées atteintes de la maladie d'Alzheimer et d'autres formes de démence [Internet]. Ottawa (Ont.) : ICIS, 2010 [consultation le 8 mai 2013]. PDF (755 Ko) téléchargeable à partir du lien : https:// secure.cihi.ca/free_products/Dementia_AIB _2010_FR.pdf
13. Conseil canadien de la santé. Aînés dans le besoin, aidants en détresse : Quelles sont les priorités de soins à domicile pour les aînés au Canada? Toronto (Ont.) : Conseil canadien de la santé, avril 2012.

14. Boustani M, Zimmerman S, Williams CS et collab. Characteristics associated with behavioral symptoms related to dementia in long-term care residents. Gerontologist. 2005;45 nº spéc. 1(1):56-61.

15. Gruber-Baldini AL, Zimmerman S, Boustani M, Watson LC, Williams CS, Reed PS. Characteristics associated with depression in long-term care residents with dementia. Gerontologist. 2005;45 Spec No 1(1):50-5.

16. Sloane PD, Zimmerman S, Gruber-Baldini AL, Hebel JR, Magaziner J, Konrad TR. Health and functional outcomes and health care utilization of persons with dementia in residential care and assisted living facilities: comparison with nursing homes. Gerontologist. 2005;45 n ${ }^{\circ}$ spéc. 1(1):124-32.

17. Hèbert R, Dubois M-F, Wolfson C, Chambers L, Cohen C. Factors associated with long-term institutionalization of older people with dementia: data from the Canadian Study of Health and Aging. J Gerontol A Biol Sci Med Sci. 2001;56(11): M693-99.

18. Lindsay J, Sykes E, McDowell I, Verreault $\mathrm{R}$, Laurin D. More than epidemiology of Alzheimer's disease: contributions of the Canadian Study of Health and Aging. Can J Psychiatry. 2004;49(2):83-91.

19. Saposnik G, Cote R, Rochon PA et collab. Care and outcomes in patients with ischemic stroke with and without preexisting dementia. Neurology. 2011;77(18): 1664-73.

20. Weintraub D, Burn DJ. Parkinson's disease: the quintessential neuropsychiatric disorder. Mov Disord. 2011;26(6):1022-31.

21. interRAI Canada. Innovations in Data, Evidence and Applications for Persons with Neurological Conditions [Internet]. Waterloo (Ont.) : University of Waterloo; [consultation le 8 mai 2013]. http://interraicanada.uwaterloo .ca/ipnc/ 
22. Institut canadien d'information sur la santé (ICIS). Système d'information sur les services à domicile (SISD) : Évaluation des incidences sur la vie privée. Ottawa (Ont.) : ICIS; 2006.

23. Hirdes JP, Ljunggren G, Morris JN, et al. Reliability of the interRAI suite of assessment instruments: a 12-country study of an integrated health information system. BMC Health Serv Res. 2008;8:277.

24. Landi F, Tua E, Onder G et collab. Minimum Data Set for home care: A valid instrument to assess frail older people living in the community. Med Care. 2000;38(12):1184-90.

25. Morris JN, Fries BE, Steel K, et al. Comprehensive clinical assessment in community setting: applicability of the MDSHC. J Am Geriatr Soc. 1997;45(8):1017-24.

26. Poss JW, Jutan NM, Hirdes, JP, et al. A review of evidence on the reliability and validity of Minimum Data Set data. Healthc Manage Forum. 2008;21(1):33-9.

27. Morris JN, Fries BE, Mehr DR, et al. MDS Cognitive Performance Scale. J Gerontol. 1994;49(4):M174-82 .

28. Morris JN, Fries BE, Morris SA. Scaling ADLs within the MDS. J Gerontol A Biol Sci Med Sci. 1999;54(11):M546-53.

29. Hirdes JP, Frijters DH, Teare GF. The MDSCHESS scale: a new measure to predict mortality in institutionalized older people. J Am Geriatr Soc. 2003;51(1):96-100.

30. Armstrong JJ, Stolee P, Hirdes JP, Poss JW. Examining three frailty conceptualizations in their ability to predict negative outcomes for home-care clients. Age Ageing. 2010; 39(6):755-8.

31. Hirdes JP, Poss JW, Curtin-Telegdi N. The Method for Assigning Priority Levels (MAPLe): a new decision-support system for allocating home care resources. BMC Med. 2008;6:9.

32. Burrows AB, Morris JN, Simon SE, Hirdes JP, Phillips C. Development of a minimum data set-based depression rating scale for use in nursing homes. Age Ageing. 2000;29(2):165-72.
33. Szczerbińska K, Hirdes JP, Zyczkowska J. Good news and bad news: depressive symptoms decline and undertreatment increases with age in home care and institutional settings. Am J Geriatr Psychiatry. 2012;20(12):1045-56.

34. Perlman CM, Hirdes JP. The Aggressive Behavior Scale: a new scale to measure aggression based on the Minimum Data Set. J Am Geriatr Soc. 2008;56(12):2298-303.

35. Hartmaier SL, Sloane PD, Guess HA, Koch GG, Mitchell CM, Phillips CD. Validation of the Minimum Data Set Cognitive Performance Scale: agreement with the Mini-Mental State Examination. J Gerontol A Biol Sci Med Sci. 1995;50(2):M128-33.

36. Hogan DB, Freiheit EA, Strain LA, et al. Comparing frailty measures in their ability to predict adverse outcome among older residents of assisted living. BMC Geriatr. 2012;12:56.

37. Mor $\mathrm{V}$, Intrator $\mathrm{O}$, Unruh $\mathrm{MA}$, Cai $\mathrm{S}$. Temporal and geographic variation in the validity and internal consistency of the Nursing Home Resident Assessment Minimum Data Set 2.0. BMC Health Serv Res. 2011;11:78

38. Wodchis WP, Naglie G, Teare GF. Validating diagnostic information on the Minimum Data Set in Ontario hospital-based long-term care. Med Care. 2008;46(8):882-7.

39. Gambassi G, Landi F, Peng L, et al. Validity of diagnostic and drug data in standardized nursing home resident assessments: potential for geriatric pharmacoepidemiology. Med Care. 1998;36(2):167-79.

40. Norton MC, Piercy KW, Rabins PV, et al. Caregiver-recipient closeness and symptom progression in Alzheimer disease. The Cache County Dementia Progression Study. J Gerontol B Psychol Sci Soc Sci. 2009; 64(5):560-8.

41. Ebly EM, Hogan DB, Rockwood K. Living alone with dementia. Dement Geriatr Cogn Disord. 1999;10(6):541-8.

42. Herrmann N, Tam DY, Balshaw R, et al., for the Canadian Outcomes Study in Dementia (COSID) Investigators. The relation between disease severity and cost of caring for patients with Alzheimer disease in Canada. Can J Psychiatry. 2010;55(12):768-75.
43. Nabalamba A, Patten SB. Prevalence of mental disorders in a Canadian household population with dementia. Can J Neurol Sci. 2010;37(2):186-94.

44. Yaffe K, Fox P, Newcomer R et collab. Patient and caregiver characteristics and nursing home placement in patients with dementia. JAMA. 2002;287(16):2090-7.

45. Kuo TC, Zhao Y, Weir S, et al. Implications of comorbidity on costs for patients with Alzheimer disease. Med Care. 2008;46(8): 839-46.

46. Landi F, Gambassi G, Lapane KL et collab. Impact of the type and severity of dementia on hospitalization and survival of the elderly. The SAGE Study Group. Dement Geriatr Cogn Disord. 1999;10(2):121-9.

47. Wolf-Klein GP, Silverstone FA, Brod MS et collab. Are Alzheimer patients healthier? J Am Geriatr Soc. 1988;36(3):219-24.

48. Zekry D, Herrmann FR, Grandjean R et collab. Demented versus non-demented very old inpatients: the same comorbidities but poorer functional and nutritional status. Age Ageing. 2008;37(1):83-9.

49. Ganguli M. A reduced risk of Alzheimer's disease in those who survive cancer. BMJ. 2012;344:e1662.

50. Lyketsos CG. Prevention of unnecessary hospitalization for patients with dementia. The role of ambulatory care. JAMA. 2012;307(2):197-8.

51. Rosenberg PB, Mielke MM, Han D et collab. The association of psychotropic medication use with the cognitive, functional, and neuropsychiatric trajectory of Alzheimer's disease. Int J Geriatr Psychiatry. 2012;27(12): 1248-57.

52. Pendlebury ST, Rothwell PM. Prevalence, incidence, and factors associated with prestroke and post-stroke dementia: a systematic review and meta-analysis. Lancet Neurol. 2009;8(11):1006-18.

53. Maxwell CJ, Vu M, Hogan DB et collab. Patterns and determinants of dementia pharmacotherapy in a population-based cohort of home care clients. Drugs Aging. 2013;30(7):569-85. 
54. Forbes DA, Markle-Reid M, Hawranik P et collab. Availability and acceptability of Canadian home and community-based services: perspectives of family caregivers of persons with dementia. Home Health Care Services Q. 2008;27(2):75-99.

55. Franz CE, Barker JC, Kim K et collab. When help becomes a hindrance: mental health referral systems as barriers to care for primary care physicians treating patients with Alzheimer's disease. Am J Geriatr Psychiatry. 2010;18(7):576-85. 